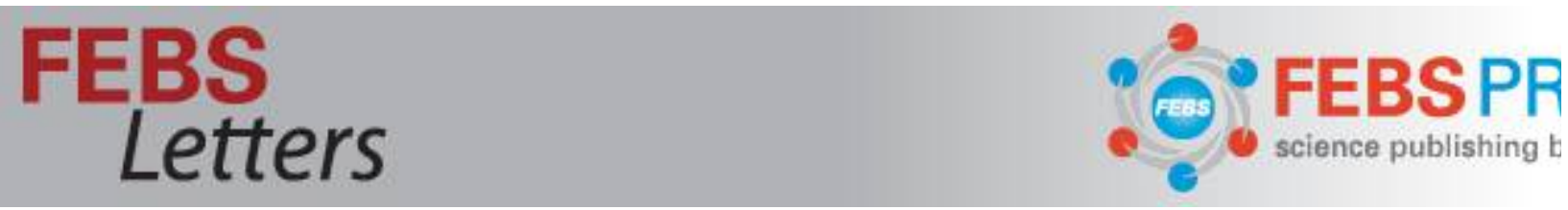

\title{
Identification of the fatty acid coenzyme-A ligase FadD1 as an interacting partner of FptX in the Pseudomonas aeruginosa pyochelin pathway
}

\begin{tabular}{|r|l|}
\hline Journal: & FEBS Letters \\
\hline Manuscript ID & Draft \\
\hline Wiley - Manuscript type: & Research Letter \\
\hline Author: & n/a \\
\hline Complete List of Authors: & $\begin{array}{l}\text { ROCHE, Béatrice; Universität Basel Department Biozentrum, Infection } \\
\text { Biology Focal Area } \\
\text { Mislin, Gaëtan; CNRS-Universite de Strasbourg, UMR7242 } \\
\text { schalk, isabelle; CNRS-Universite de Strasbourg, UMR7242 }\end{array}$ \\
\hline Keywords: & $\begin{array}{l}\text { Pseudomonas aeruginosa, pyochelin siderophore, iron uptake, } \\
\text { coenzyme-A ligase, membrane complex }\end{array}$ \\
\hline Abstract: & $\begin{array}{l}\text { Pseudomonas aeruginosa is one of the most important nosocomial } \\
\text { bacteria emerging as a highly multidrug-resistant pathogen. P. } \\
\text { aeruginosa produces two siderophores including pyochelin to fulfill its } \\
\text { need for iron during infections. We know that both outer and inner } \\
\text { membrane proteins FptA and FptX are involved in the ferri-pyochelin } \\
\text { uptake, but this process requires increasing molecular and biochemical } \\
\text { knowledge. Here, using bacterial two-hybrid and copurification assays we } \\
\text { identified the fatty acid coenzyme-A ligase FadD1 as a novel interacting } \\
\text { partner of the inner membrane transporter FptX, and found that FadD1 } \\
\text { may play a role in pyochelin production. We managed to purify the } \\
\text { FadD1-FptX inner membrane complex and obtained low-resolution 3D } \\
\text { models, opening the way for future high-resolution structures. }\end{array}$ \\
\hline
\end{tabular}


1 Identification of the fatty acid coenzyme-A ligase FadD1 as an interacting partner of FptX in the

$2 \quad$ Pseudomonas aeruginosa pyochelin pathway

4 Béatrice Roche ${ }^{1,2,3^{*}}$, Gaëtan L.A. Mislin ${ }^{1,2}$ and Isabelle J. Schalk ${ }^{1,2}$

5 1. CNRS, UMR7242, ESBS, Bld Sébastien Brant, F-67412 Illkirch, France.

6 2. Université de Strasbourg, UMR7242, ESBS, Bld Sébastien Brant, F-67412 Illkirch, France.

7 3. Present address: Biozentrum, University of Basel, CH-4056 Basel, Switzerland

$8 * *$ correspondence should be addressed to Béatrice Roche (beatrice.roche@unibas.ch)

Abstract

Pseudomonas aeruginosa is one of the most important nosocomial bacteria emerging as a highly

12 multidrug-resistant pathogen. $P$. aeruginosa produces two siderophores including pyochelin to fulfill its

13 need for iron during infections. We know that both outer and inner membrane proteins FptA and FptX are

14 involved in the ferri-pyochelin uptake, but this process requires increasing molecular and biochemical

15 knowledge. Here, using bacterial two-hybrid and copurification assays we identified the fatty acid

16 coenzyme-A ligase FadD1 as a novel interacting partner of the inner membrane transporter FptX, and found

17 that FadD1 may play a role in pyochelin production. We managed to purify the FadD1-FptX inner

18 membrane complex and obtained low-resolution 3D models, opening the way for future high-resolution

19 structures.

22 Keywords: Pseudomonas aeruginosa; pyochelin siderophore; iron uptake; coenzyme-A ligase; membrane 23 complex. 


\section{Introduction}

28 Pseudomonas aeruginosa is a major human pathogen naturally resistant to many antibiotics due to the low 29 permeability of its envelope. The situation is particularly critical as $P$. aeruginosa belongs to the World 30 Health Organization list of antibiotic-resistant priority pathogen [1]. The success of bacteria to colonize the

31 host relies mostly on their ability to obtain adequate supplies of the nutrients that are essential for growth 32 and virulence. To circumvent the low bioavailability of iron in the host during infections, $P$. aeruginosa 33 produces the two siderophores pyoverdine (PVD) and pyochelin (PCH) [2-4]. Siderophores are small iron

34 chelating compounds produced by bacteria under iron limitation conditions [5]. Previous studies have shown

35 an upregulation of the PCH pathway in clinical isolates from cystic fibrosis patients [6-7] and reported a

36 potential role of $\mathrm{PCH}$ in the inflammatory response [8].

$37 \quad \mathrm{PCH}$ is synthesized by non-ribosomal peptide synthetases in the bacterial cytoplasm and then secreted into

38 the extracellular medium by an unknown mechanism [9]. Once in the bacterial environment, $\mathrm{PCH}$ chelates

39 ferric iron leading to the formation of ferri-PCH complexes. Only two proteins involved in the ferri-PCH

40 uptake have been described so far: the specific TonB-dependent outer membrane transporter FptA [10-12],

41 and the inner membrane protein FptX [13-15]. Much attention has been paid to structural studies of FptA,

42 and X-ray structure of FptA bound to ferri-PCH provided valuable information for the design of $\mathrm{PCH}-$

43 antibiotic conjugates [16-17]. In the context of antibiotic resistance, conjugation of antibiotics with

44 siderophore is a promising drug delivery strategy exploiting iron uptake systems as gates to carry out

45 antibiotics into the bacteria [18-19]. Such Trojan horse strategy can provide increased penetration of drugs

46 previously unable to cross the cell wall of bacteria [4]. But we currently lack biochemical/structural data on

47 the inner membrane transporter FptX predicted to have 12 transmembrane segments.

48 Here, we explored potential new actors involved in the ferri-PCH uptake pathway by using a genome-wide

49 screening approach. We identified the fatty acid coenzyme-A ligase FadD1 as a new and previously

50 undescribed interacting partner of FptX, forming an inner membrane complex. Deletion of fadD1 led to a

51 decrease in PCH production, suggesting a role of FadD1 in biosynthesis and/or secretion of this siderophore. 
52 We also managed to express and purify FptX and could establish low-resolution 3D models of FptX, FadD1

53 and of the FadD1-FptX complex by negative-stain electron microscopy (EM).

\section{$55 \quad$ Materials and methods}

\section{Chemicals and growth media.}

57 X-Gal (5-bromo-4-chloro-3-indoyl- $\beta$-D-galactopyranoside) and IPTG (Isopropyl- $\beta$-D thiogalactoside) were 58 purchased from Euromedex. ONPG (2-Nitrophenyl- $\beta$-D galactopyranoside), sodium oleate, and N59 lauroylsarcosine sodium salt were obtained from Sigma-Aldrich. Detergent n-Dodecyl- $\beta$-D60 maltopyranoside (DDM) was purchased from Anatrace. LB (Lennox) and LB agar medium were both

61 purchased from Difco and were used as nutriment-rich medium in all experiments. Bacteria were routinely 62 grown at $30^{\circ} \mathrm{C}$ in LB broth (Difco) or in iron-restricted medium (CAA) as previously described [15]. Media 63 were supplemented as necessary with ampicillin (Ap; $100 \mu \mathrm{g} \cdot \mathrm{mL}^{-1}$ ), kanamycin (Kan; $50 \mu \mathrm{g} \cdot \mathrm{mL}^{-1}$ ), 64 gentamicin $(\mathrm{Gm} ; 30 \mu \mathrm{g} \cdot \mathrm{mL}-1)$, chloramphenicol $\left(\mathrm{Cm} ; 50 \mu \mathrm{g} \cdot \mathrm{mL}^{-1}\right)$, IPTG $(0.5 \mathrm{mM})$. The strains used in this 65 study are listed in Table S1.

Plasmids construction.

68 All DNA amplifications for cloning were performed with Phusion High-Fidelity DNA polymerase

69 (ThermoFisher Scientific) using genomic DNA of PAO1 strain as template. Restriction enzymes and T4

70 DNA ligase were purchased from ThermoFisher Scientific. All enzymes were used in accordance with the 71 manufacturer's instructions. Oligonucleotides are listed in Table S2. Escherichia coli strain Top10 was used 72 as the host strain for all plasmids. Absence of mutations was checked by DNA sequencing (GATC).

73 Expression plasmids. Plasmid p33-fptX $\left(\mathrm{His}_{6}\right)$ was constructed by PCR amplification of the coding region

74 of $f p t X$ by using the primer pairs 970/969. The PCR product was then digested with EcoRI and HindIII

75 enzymes and ligated into the EcoRI/HindIII linearized pBAD33 vector. Plasmid p24-fadD1(StrepII) was

76 constructed by PCR amplification of the coding region of fadDl by using the primer pairs $1157 / 1158$. The 
77 PCR product was then digested with NcoI and HindIII enzymes and ligated into the NcoI/HindIII linearized 78 pBAD24 vector.

79 Two-hybrid plasmids. DNA fragments encoding proteins of interest were fused to the two fragments T25

80 and T18 of Bordetella pertussis adenylate cyclase carried on the pKT25, pKNT25, pUT18C and pUT18

81 vectors. The DNA regions encoding $f p t X$ and $f a d D 1$ were amplified by PCR by using primer pairs $951 / 952$

82 and 1037/1038, respectively. PCR products were then digested with XbaI and KpnI enzymes and ligated 83 into the XbaI/KpnI linearized pKT25, pKNT25, pUT18C and pUT18 vectors.

84

\section{Bacterial two-hybrid screen.}

86 Four independent two-hybrid libraries (pUT18CPAO1Lib, pUT18PAO1Lib, pUT18+1PAO1Lib and 87 pUT18+2PAO1Lib) of PAO1 genome were tested independently. The pKNT25-fptX vector was used as 88 the bait and the two-hybrid screen was performed as described in Houot et al., 2012 [20].

\section{Bacterial two-hybrid assay.}

91 The adenylate cyclase-based bacterial two-hybrid technique was used as previously published [21].

\section{Western blot analysis.}

94 Proteins were applied to SDS-PAGE acrylamide gels and transferred onto nitrocellulose membranes. $95 \quad \mathrm{FptX}\left(\mathrm{His}_{6}\right)$ and FadD1(StrepII) proteins were immunodetected with the anti-His $(1: 3,000$; GeneTex $)$ and 96 the StrepMAB $(1: 1,000$; IBA) antibodies, respectively. Immunoblots were developed by using horseradish 97 peroxidase-conjugated goat anti-rabbit and anti-mouse antibodies (1:10,000; GE Healthcare), followed by 98 chemiluminescence detection.

\section{Expression and purification of FptX.}

101 Top10 E. coli cells were transformed with the $\mathrm{p} 33-\mathrm{fptX}\left(\mathrm{His}_{6}\right)$ vector. Cells were grown at $37^{\circ} \mathrm{C}$ with shaking 102 until they reached an $\mathrm{OD}_{600} \approx 0.5$, and the expression of $f p t X$ gene was induced with $0.01 \%$ arabinose for 
$1034 \mathrm{~h}$ at $37^{\circ} \mathrm{C}$. Cell pellets were resuspended in $50 \mathrm{mM}$ Tris- $\mathrm{HCl} \mathrm{pH} 8,100 \mathrm{mM} \mathrm{NaCl}$, supplemented with 104 EDTA-free protease inhibitor (Roche). After sonication, the cell suspension was clarified by centrifugation 105 at 7,700 $\mathrm{g}$ for $15 \mathrm{~min}$ at $4^{\circ} \mathrm{C}$. Membrane fraction was then collected by ultracentrifugation at $118,000 \mathrm{~g}$ for $10640 \mathrm{~min}$ at $4^{\circ} \mathrm{C}$. After mechanically homogenized, membranes were solubilized in $50 \mathrm{mM}$ Tris- $\mathrm{HCl} \mathrm{pH} 8$, $107200 \mathrm{mM} \mathrm{NaCl}, 1 \% \mathrm{DDM}$ at $4^{\circ} \mathrm{C}$ overnight. The suspension was clarified by ultracentrifugation at $108118,000 \mathrm{~g}$ for $40 \mathrm{~min}$ at $4^{\circ} \mathrm{C}$. The supernatant was loaded onto a 1-mL HisTrap HP column (GE Healthcare) 109 equilibrated with buffer A (50 mM Tris- $\mathrm{HCl} \mathrm{pH}$ 8, $500 \mathrm{mM} \mathrm{NaCl}, 0.1 \%$ n-Dodecyl- $\beta$-D-maltopyranoside 110 (DDM), $20 \mathrm{mM}$ imidazole). FptX was eluted in buffer A containing $500 \mathrm{mM}$ imidazole. Central fraction of

111 the peak was loaded onto a Superdex 200 10/300 GL column (GE Healthcare) equilibrated with $50 \mathrm{mM}$

112 Hepes $\mathrm{pH} 7.5,50 \mathrm{mM} \mathrm{NaCl}, 0.025 \%$ DDM. FptX was eluted as a single peak and the central fraction was 113 immediately used for EM sample preparation.

115 Expression and purification of FadD1.

116 Top10 E. coli cells were transformed with the p24-fadD1(StrepII) vector. Cell growth, gene induction and 117 membrane preparation were done as described for FptX. The supernatant was loaded onto a 5-mL StrepTrap 118 HP column (GE Healthcare) equilibrated with buffer A (50 mM Tris-HCl pH 8, $200 \mathrm{mM} \mathrm{NaCl}, 0.1 \%$ 119 DDM). FadD1 was eluted in buffer A containing $2.5 \mathrm{mM}$ D-Desthiobiotin. Peak fractions were pooled and 120 loaded onto a Superdex 200 10/300 GL (GE Healthcare) column equilibrated with $50 \mathrm{mM}$ Hepes pH 7.5, $12150 \mathrm{mM} \mathrm{NaCl}, 0.025 \%$ DDM. FadD1 was eluted as a single peak and the central fraction was immediately 122 used for EM sample preparation.

\section{Expression and purification of the FadD1-FptX complex.}

125 Top10 E. coli cells were transformed with the $\mathrm{p} 33-\mathrm{fptX}\left(\mathrm{His}_{6}\right)$ and $\mathrm{p} 24-\mathrm{fadD} 1$ (StrepII) vectors. Cell growth, 126 gene induction and membrane preparation were done as described for FptX. The supernatant was loaded 127 onto a 1-mL StrepTrap HP column (GE Healthcare) equilibrated with buffer A (50 mM Tris- $\mathrm{HCl} \mathrm{pH} 8$, $12850 \mathrm{mM} \mathrm{NaCl}, 0.1 \%$ DDM). The FadD1-FptX complex was eluted in buffer A supplemented with $2.5 \mathrm{mM}$ 
129 D-Desthiobiotin (Sigma Aldrich) into a 1-mL HisTrap HP column (GE Healthcare). The column was

130 washed in buffer A and the FadD1-FptX complex was eluted in buffer A supplemented with $250 \mathrm{mM}$

131 imidazole. Central fraction of the peak was then loaded onto a Superdex 200 column 10/300 GL (GE

132 Healthcare) equilibrated with $50 \mathrm{mM}$ Tris- $\mathrm{HCl} \mathrm{pH}$ 8, $50 \mathrm{mM} \mathrm{NaCl}, 0.025 \%$ DDM. The FadD1-FptX

133 complex was eluted as a single peak, and the central fraction was immediately used for EM sample

134 preparation.

136 EM processing of FptX, FadD1 and the FadD1-FptX complex.

137 Determination of FadD1-FptX complex, FptX and FadD1 proteins structure was made by negative-stain

138 EM. $5 \mu \mathrm{L}$ of FadD1-FptX complex (28 ng. $\left.\mu \mathrm{L}^{-1}\right)$, FptX $\left(23\right.$ ng. $\left.\mu \mathrm{L}^{-1}\right)$ and FadD1 $\left(6.25\right.$ ng. $\left.\mu \mathrm{L}^{-1}\right)$ sample was

139 fixed with $0.6 \mu \mathrm{L}$ glutaraldehyde $1 \%$. The sample was then spotted to 300 meshs $\mathrm{Cu}$ grids covered with a

140 carbon film (Euromedex CF300-CU-050). After 60s of absorption, each sample was stained with 2\% uranyl

141 acetate. For the FadD1-FptX complex, the sample was washed with one drop of $50 \mathrm{mM}$ Tris $\mathrm{pH} 8,50 \mathrm{mM}$

$142 \mathrm{NaCl}$ before staining with $2 \%$ uranyl acetate. Images were recorded automatically using the SerialEM

143 software on a FEI Tecnai F20 microscope operating at a voltage of $200 \mathrm{kV}$ and a defocus range of $500 \mathrm{~nm}$,

144 using a Gatan US1000 detector at a nominal magnification of 50,000X, yielding a pixel size of 2.12. A dose

145 rate of 20 electrons per square angstrom per second, and an exposure time of 10 s were used. For the FadD1-

146 FptX complex, a total of 58,034 particles were selected from 3,000 independent images and extracted within

147 boxes of 200 pixels using Relion2. For FptX, a total of 38,746 particles were selected from 1,500

148 independent images and extracted within boxes of 192 pixels using Relion2. For FadD1, a total of 102,978

149 particles were selected from 1,500 independent images and extracted within boxes of 120 pixels using

150 Relion2. The defocus value was estimated, and the contrast transfer function was corrected using CTF

151 estimation in Relion2. We used two rounds of reference-free 2D class averaging to clean up the selected

152 data set. High-populated classes displaying high-resolution features were conserved during the procedure

153 with a final data set of 30,355 particles for the FadD1-FptX complex, 33,001 particles for FptX and 61,553

154 particles for FadD1. An initial 3D model was generated in EMAN2. Relion2 auto-refine procedure was used 
155 to obtain a final reconstruction at $33-\AA \AA, 26-\AA$ and $17-\AA$ resolution for FadD1-FptX, FptX and FadD1, 156 respectively, after masking and without symmetry constraints. Resolutions are based on the "gold standard" 157 Fourier shell correlation (FSC) (Fig. S3). USCF Chimera was used to display three-dimensional 158 reconstructions.

\section{Iron uptake assays and cell fractionation.}

161 Cells were cultured overnight in LB medium at $30^{\circ} \mathrm{C}$, and afterwards at $30^{\circ} \mathrm{C}$ overnight in CAA medium.

162 Then, they were diluted in fresh CAA medium and grown at $30^{\circ} \mathrm{C}$ overnight. Bacteria were washed with $16350 \mathrm{mM}$ Tris- $\mathrm{HCl}$ at $\mathrm{pH} 8$ and diluted to an $\mathrm{OD}_{600 \mathrm{~nm}}$ of 1 . The $\mathrm{PCH}-{ }^{55} \mathrm{Fe}$ complex was prepared as previously 164 described [22]. Cells were incubated $30 \mathrm{~min}$ with $200 \mathrm{nM} \mathrm{PCH}-{ }^{55} \mathrm{Fe}$ at $30^{\circ} \mathrm{C}$. After incubation, cells were 165 centrifuged for $8 \mathrm{~min}$ at $6,700 \mathrm{~g}$ to obtain the supernatant as the extracellular medium, and the cell pellet 166 which was resuspend in Tris-sucrose EDTA (Tris-HCl $0.2 \mathrm{M} \mathrm{pH} \mathrm{8,} \mathrm{EDTA} 1 \mathrm{mM}$, sucrose $20 \%$ ).

167 Spheroplasts were obtained by adding $200 \mu \mathrm{g} \cdot \mathrm{mL}^{-1}$ lysozyme (Euromedex) to the suspension and incubated

168 at $4^{\circ} \mathrm{C}$ for $1 \mathrm{~h}$. The suspension was centrifuged for $8 \mathrm{~min}$ at $6,700 \mathrm{~g}$ at $4^{\circ} \mathrm{C}$ to obtain the spheroplast pellet 169 and the periplasmic fraction (supernatant). The supernatant was ultracentrifuged $\left(120,000 \mathrm{~g}, 40 \mathrm{~min}, 4^{\circ} \mathrm{C}\right)$ 170 to clarify the periplasmic fraction. Spheroplast pellet was washed with Tris- $\mathrm{HCl} 0.2 \mathrm{M} \mathrm{pH} \mathrm{8,} \mathrm{sucrose} 20 \%$, 171 and resuspend in cold water by vortexing. The suspension was then incubated with benzonase for $1721 \mathrm{~h}$ at $37^{\circ} \mathrm{C}$. The cytoplasmic fraction was isolated by ultracentrifugation $\left(120,000 \mathrm{~g}, 40 \mathrm{~min}, 4^{\circ} \mathrm{C}\right)$. Pellet 173 was washed twice with cold water and resuspended in Tris- $\mathrm{HCl} 0.2 \mathrm{M} \mathrm{pH} \mathrm{8,} \mathrm{sucrose} 20 \%$ to obtain the 174 bacterial membranes.

\section{Deletion mutant construction.}

177 For the deletion of both $f a d D 1$ and $f a d D 2$ genes, PCR was used to generate a 500 bp DNA fragment 178 upstream of $f a d D 2$ and a 500 bp DNA fragment downstream of $f a d D 1$, by using the primer pairs 1280/1281

179 and 1282/1151, respectively. Each PCR product was linked by overlapping PCR and products were digested 180 with HindIII and EcoRI and cloned into the suicide vector pEXG2, yielding pEXG2 $\Delta$ fadD1 $\Delta$ fadD2. 
181 Deletion in the chromosomal genome of $P$. aeruginosa was generated by transferring the suicide vector 182 from E. coli SM10 strains into recipient strains allowing the plasmid to integrate into the chromosome, with 183 selection for gentamicin resistance. A second crossing-over event excising the vector was selected using $184 \mathrm{NaCl}$-free LB plates supplemented with $5 \%$ sucrose. Deletions were confirmed by sequencing using 185 external primers.

\section{PCH production.}

188 Cells were cultured overnight in $\mathrm{LB}$ medium at $37^{\circ} \mathrm{C}$, and afterwards at $30^{\circ} \mathrm{C}$ overnight in iron-deficient 189 CAA medium. They were then diluted to $0.1 \mathrm{OD}_{600 \mathrm{~nm}}$ units in fresh CAA medium and incubated at $30^{\circ} \mathrm{C}$ for $19016 \mathrm{~h}$. The extracellular medium was separated from the cells by centrifugation at 7,700 $\mathrm{g}$ for $15 \mathrm{~min}$. The $191 \mathrm{pH}$ of the supernatant was acidified by adding $1 \mathrm{M}$ citric acid and PCH was extracted with dichloromethane. 192 Pyochelin concentration was determined by measuring the absorbance at $320 \mathrm{~nm}\left(\varepsilon=4,300 \mathrm{~L} \cdot \mathrm{mol}^{-1} 1 . \mathrm{cm}^{-1}\right)$. 193 Results are expressed as a function of $\mathrm{PCH}$ produced $(\mu \mathrm{M})$ to bacterial growth $\left(\mathrm{OD}_{600 \mathrm{~nm}}\right)$.

\section{Significance tests}

196 Student's $t$ test was used for two-group comparisons using unpaired two-tailed $t$ test. Analyses were 197 performed using GraphPad Prism 8.

\section{Results}

201 Identification of FptX interacting partners. FptX is proposed as the inner membrane protein involved in 202 ferri-PCH uptake in $P$. aeruginosa [13-15]. In order to find other proteins involved in this pathway, we 203 looked for FptX-interacting partners by using a bacterial two-hybrid genome wide screening. The pKNT25204 fptX bait plasmid was combined with the P. aeruginosa PAO1 fragment prey libraries constructed in the 205 pUT18 vector by Houot et al., 2012 [20]. Blue colonies reflecting a protein interaction with FptX were identified, and the DNA sequence inserts contained in the pUT18 derivative vectors were further sequenced. 
207 The prey fragments corresponded either to the 253 first amino acids of the fatty acid coenzyme-A ligase 208 FadD1, or to the 120 first amino acids of PA1316, a putative major facilitator superfamily transporter. Only 209 FadD1 was identified multiple times throughout this screen and the interaction between FadD1 and FptX 210 was validated by cloning the entire proteins in two-hybrid vectors (Fig. 1A). The interaction was also 211 confirmed by co-expressing FadD1 with a StrepII tag and FptX with a His 6 tag at the carboxy terminus in 212 E. coli. Total membranes were isolated and solubilized using the detergent DDM. The complex was 213 specifically isolated by a two-step affinity chromatography and the presence of FadD1(StrepII) (63 kDa) 214 and $\mathrm{FptX}\left(\mathrm{His}_{6}\right)(44 \mathrm{kDa})$ was confirmed by immunoblot analysis (Fig. 1B and C). The overall FptX(His 6 ) 215 distribution on SDS-PAGE reveals several bands ranging from 44 to $150 \mathrm{kDa}$, suggesting multimerization 216 of the protein. Altogether, these results revealed a new complex between the inner membrane transporter 217 FptX and the fatty acid coenzyme-A ligase FadD1.

219 FadD1 is involved in PCH production rather than ferri-PCH uptake. This interaction between FptX 220 and FadD1 must involve a biological role for FadD1 in the $\mathrm{PCH}$ pathway. Based on the role of FptX in ferri221 PCH uptake [15], we first investigated the possible involvement of FadD1 in iron uptake via PCH. 222 P. aeruginosa has in its genome two genes encoding closely related enzymes for the fatty acid metabolism, 223 FadD1 and FadD2, which exhibit $60 \%$ identity [23]. We deleted the two fadD genes to avoid any functional 224 redundancy. $\mathrm{PCH}-{ }^{55} \mathrm{Fe}$ uptake assays were performed using a $P$. aeruginosa strain unable to produce PVD 225 and $\mathrm{PCH}(\Delta p v d F \Delta p c h A)$ in order to control the PCH concentration and to have no iron uptake by PVD, the 226 other siderophore produced by $P$. aeruginosa. Cells were grown under iron-restricted conditions, harvested 227 and incubated in the presence of $200 \mathrm{nM} \mathrm{PCH}-{ }^{55} \mathrm{Fe}$. After $30 \mathrm{~min}$ incubation, cells were pelleted and the 228 cytoplasmic, periplasmic and membrane fractions isolated by cell fractionation and the radioactivity 229 monitored in each cell compartment (Fig. 2).

230 In the $\triangle p v d F \Delta p c h A$ control strain, most of the radioactivity (53\%) was found in the cytoplasm, $8 \%$ in the 231 periplasm, $18 \%$ associated to the membranes and $21 \%$ still in the extracellular medium (Fig. 2), while for 232 the $\Delta f p t X$ mutant most of ${ }^{55} \mathrm{Fe}$ was not imported into the bacteria and found in the extracellular medium 
233 (Fig. 2). In the absence of FptX, the ferri-PCH complex cannot enter the cells, which is related to previous 234 data showing that the expression of $f p t A$, encoding for the outer membrane transporter, is drastically 235 decreased in an $\Delta f p t X$ mutant [24]. Regarding the $\Delta f a d D 1 D 2$ mutant, a very similar ${ }^{55} \mathrm{Fe}$ repartition was 236 observed compare to the control strain, indicating that FadD1 plays no role in iron acquisition by $\mathrm{PCH}$.

237 We next investigated the effect of fadD1D2 mutations on $\mathrm{PCH}$ production. WT and the corresponding 238 mutant strains were grown under iron-starvation conditions and PCH was extracted from growth medium 239 and its concentration determined using its characteristic absorbance at $320 \mathrm{~nm}$. A 1.4-, 2.4-, and 4.4-fold 240 decrease in $\mathrm{PCH}$ concentration in the growth medium was observed for the $\Delta f a d D 1 D 2, \Delta f p t X$ and $241 \Delta f p t X \triangle f a d D 1 D 2$ mutants respectively, compared to the wild type PAO1 strain (Fig. 3), suggesting a role of 242 FadD1 in PCH production and/or secretion.

\section{Biochemical and architectural analysis of FptX, FadD1 and the FadD1-FptX complex.}

245 In contrast to the available X-ray structure of the outer membrane transporter FptA [11], structural data are 246 still lacking for the inner membrane transporter FptX. The determination of the 3D structure of this polytopic 247 inner membrane protein remains very challenging because of the low expression levels of FptX even in 248 overproducing bacterial systems. Consequently, we then investigated the architecture of FptX, FadD1 and 249 the FadD1-FptX complex by negative-stain EM. First, the proteins were produced and purified from E. coli 250 membranes and their functional integrity was established by assessing the binding activity to ferri-PCH for 251 FptX and the FadD1-FptX complex, and the fatty acid coenzyme-A ligase activity for FadD1 (Fig. S1). EM 252 data set were collected, and reference-free two-dimensional class revealed characteristic views of the 253 FadD1-FptX complex, FptX and FadD1 (Fig. S2). The EM data set was used to reconstruct a 33- $\AA$, 26- $\AA$ 254 and 17-Å resolution three-dimensional (3D) model of the FadD1-FptX complex, FptX and FadD1 proteins, 255 respectively (Fig. 4). FadD1-FptX complex is $\sim 230 \AA \AA$ in height and $\sim 150 \AA$ in diameter. FptX is $\sim 225 \AA$ in 256 height and $\sim 120 \AA$ in diameter, and FadD1 is $\sim 115 \AA$ in height and $\sim 90 \AA$ in diameter. The FptX EM model 257 was then docked into the EM volume of the FadD1-FptX complex, showing that FptX occupies most of the 258 EM envelope of the complex (Fig. 4). However, at this low resolution and according to the small volume of 
259 FadD1, the electron density may not have sufficiently distinctive features for an unambiguous placement of 260 FadD1 in the EM volume complex.

\section{Discussion}

263 The data presented here give new insights in the P. aeruginosa PCH siderophore pathway. Previous studies 264 had shown that FptA and FptX are the outer and inner membrane transporters involved in iron uptake by 265 the siderophore $\mathrm{PCH}[10-15]$. Both proteins also play a role in $\mathrm{PCH}$ production: in $\Delta f p t A$ and $\Delta f p t X$ mutants 266 no more $\mathrm{PCH}-\mathrm{Fe}$ is transported into bacteria, affecting the $\mathrm{PCH}-$ mediated signaling cascade, and hence $\mathrm{PCH}$ 267 production $[14,24]$.

268 Here, the bacterial two-hybrid screen represented a powerful tool as it identified for the first time a fatty 269 acid coenzyme-A ligase enzyme, FadD1, as an interacting partner of the inner membrane transporter FptX. 270 FadD1 is described as a fatty acid coenzyme-A ligase involved in fatty acid metabolism in P. aeruginosa 271 [23]. To assess the physiological significance of the FadD1-FptX complex, we explored the impact of FadD1

272 on both ferri-PCH uptake and PCH production. We showed that FadD1 has no impact on iron uptake by $273 \mathrm{PCH}$ but is required for optimum PCH production. FadD1 may play a role in biosynthesis and/or secretion 274 of $\mathrm{PCH}$, but the data do not allow to discriminate between these two biological processes. Our findings 275 suggest a potential acylation of PCH. FadD1 might load a fatty acid chain to PCH, allowing PCH to be 276 anchored to the inner membrane during its synthesis as described previously for PVD, ferrichrome or 277 mycobactin [25-27]. For mycobactin, the fatty acid ligase FadD33 (also known as MbtM) was reported to 278 transfer a lipophilic aliphatic chain to the $\epsilon$-amino group of the lysine mycobatin core [27]. In the case of 279 PVD, the biosynthesis begins with an acylation introducing a myristic or myristoleic group carried out by 280 the first module of the PvdL non-ribosomal peptide synthetase [26]. The presence of a myristic or 281 myristoleic acid group retains the PVD precursor at the membrane during its biosynthesis. For both PVD 282 and mycobactin, the acylation of siderophore promotes plasma membrane localization. Further experiments 283 are still necessary to identify the role of an acylation of PCH by FadD1. Moreover, currently nothing is 284 known concerning the proteins and the molecular mechanisms involved in PCH secretion as well as in the 
285 molecular mechanism allowing iron release from $\mathrm{PCH}$.

286 FptX was reported to be a homolog of the transporter of rhizobactin $\mathrm{RhtX}$, belonging to the major facilitator 287 superfamily (MFS) transporters [13]. Currently, structure determination of such inner membrane proteins 288 remains a difficult task and presents a challenge for expression and purification. Here, we established for 289 the first time a low-resolution 3D model of FptX, its interacting partner FadD1 and of the FadD1-FptX 290 complex by negative-stain EM. Due to the lack of information concerning the various domains of FptX and 291 the low resolution of the EM reconstruction, it was impossible to clearly define a topology of the complex. 292 Altogether, these results highlight a new actor in the PCH pathway and suggest that this pathway would 293 probably be more complex than what has been described so far. In addition, our efforts to purify and initiate 294 low resolution EM-models pave the way for future high-resolution studies for structure-based drug design 295 to counter antibiotic resistance, one of the biggest public health challenges of our time.

\section{Acknowledgement}

298 Authors acknowledge the Centre National de la Recherche Scientifique (CNRS) for general financial 299 support. BR would like to thank Roche Pharmaceutical Research and Early Development Basel for their 300 financial support via the Roche Postdoctoral Fellowship (RPF) Program. We acknowledge the support and 301 the use of resources of the French Infrastructure for Integrated Structural Biology (FRISBI) ANR-10-INBS302 05, "Project ID 52", and of Instruct-ERIC, "PID 2886". Specifically, we would like to thank Christine 303 Ruhlmann and Corinne Crucifix (IGBMC, Strasbourg) for help with EM sample preparation and EM data 304 collection, and Veronique Mallouh (IGBMC, Strasbourg) for help with data processing. We acknowledge 305 Eric Durand (LISM, Marseille) for fruitful EM processing discussion. We acknowledge Christophe Bordi 306 (LISM, Marseille), Laetitia Houot (LISM, Marseille) and Aurélia Battesti (LCB, Marseille) for kindly 307 providing the PAO1 two-hybrid libraries and the two-hybrid vectors, and Laurent Loiseau (LCB, Marseille) 308 for providing pBAD vectors. 


\section{Author contributions}

312 BR and IS designed the experiments. BR performed the experiments. GM provided the PCH siderophore.

313 BR wrote the manuscript. IS helped to improve the article. All authors discussed and commented on the 314 manuscript.

\section{References}

3171 Tommasi R, Brown DG, Walkup GK, Manchester JI \& Miller AA (2015) ESKAPEing the labyrinth of 318 antibacterial discovery. Nat Rev Drug Discov 14, 529-542.

3192 Cox CD, Rinehart KL, Moore ML \& Cook JC (1981) Pyochelin: novel structure of an iron-chelating 320 growth promoter for Pseudomonas aeruginosa. Proc Natl Acad Sci U S A 78, 4256-4260.

3213 Thomas MS (2007) Iron acquisition mechanisms of the Burkholderia cepacia complex. Biometals Int J

322 Role Met Ions Biol Biochem Med 20, 431-452.

3234 Schalk IJ (2018) Siderophore-antibiotic conjugates: exploiting iron uptake to deliver drugs into bacteria.

324 Clin Microbiol Infect Off Publ Eur Soc Clin Microbiol Infect Dis 24, 801-802.

3255 Hider RC \& Kong X (2010) Chemistry and biology of siderophores. Nat Prod Rep 27, 637-657.

3266 Hare NJ, Soe CZ, Rose B, Harbour C, Codd R, Manos J \& Cordwell SJ (2012) Proteomics of

327 Pseudomonas aeruginosa Australian epidemic strain 1 (AES-1) cultured under conditions mimicking the

328 cystic fibrosis lung reveals increased iron acquisition via the siderophore pyochelin. J Proteome Res 11, $329776-795$.

3307 Rossi E, Falcone M, Molin S \& Johansen HK (2018) High-resolution in situ transcriptomics of

331 Pseudomonas aeruginosa unveils genotype independent patho-phenotypes in cystic fibrosis lungs. Nat

332 Commun 9, 3459.

3338 Lyczak JB, Cannon CL \& Pier GB (2002) Lung infections associated with cystic fibrosis. Clin

334 Microbiol Rev 15, 194-222.

3359 Reimmann C, Serino L, Beyeler M \& Haas D (1998) Dihydroaeruginoic acid synthetase and pyochelin

336 synthetase, products of the pchEF genes, are induced by extracellular pyochelin in Pseudomonas 
aeruginosa. Microbiol Read Engl 144 ( Pt 11), 3135-3148.

33810 Ankenbauer RG (1992) Cloning of the outer membrane high-affinity Fe(III)-pyochelin receptor of

339 Pseudomonas aeruginosa. J Bacteriol 174, 4401-4409.

34011 Cobessi D, Celia H \& Pattus F (2005) Crystal structure at high resolution of ferric-pyochelin and its

341 membrane receptor FptA from Pseudomonas aeruginosa. J Mol Biol 352, 893-904.

34212 Mislin GLA, Hoegy F, Cobessi D, Poole K, Rognan D \& Schalk IJ (2006) Binding properties of

343 pyochelin and structurally related molecules to FptA of Pseudomonas aeruginosa. J Mol Biol 357, 1437-

3441448.

34513 Cuív PO, Clarke P, Lynch D \& O’Connell M (2004) Identification of rhtX and fptX, novel genes

346 encoding proteins that show homology and function in the utilization of the siderophores rhizobactin 1021

347 by Sinorhizobium meliloti and pyochelin by Pseudomonas aeruginosa, respectively. J Bacteriol 186,

$348 \quad 2996-3005$.

34914 Michel L, Bachelard A \& Reimmann C (2007) Ferripyochelin uptake genes are involved in pyochelin350 mediated signalling in Pseudomonas aeruginosa. Microbiol Read Engl 153, 1508-1518.

35115 Cunrath O, Gasser V, Hoegy F, Reimmann C, Guillon L \& Schalk IJ (2015) A cell biological view of 352 the siderophore pyochelin iron uptake pathway in Pseudomonas aeruginosa. Environ Microbiol 17, 171353185.

35416 Noël S, Gasser V, Pesset B, Hoegy F, Rognan D, Schalk IJ \& Mislin GLA (2011) Synthesis and 355 biological properties of conjugates between fluoroquinolones and a N3' '-functionalized pyochelin. Org 356 Biomol Chem 9, 8288-8300.

35717 Paulen A, Hoegy F, Roche B, Schalk IJ \& Mislin GLA (2017) Synthesis of conjugates between 358 oxazolidinone antibiotics and a pyochelin analogue. Bioorg Med Chem Lett 27, 4867-4870.

35918 Ghosh M, Miller PA, Möllmann U, Claypool WD, Schroeder VA, Wolter WR, Suckow M, Yu H, Li S, 360 Huang W, Zajicek J \& Miller MJ (2017) Targeted Antibiotic Delivery: Selective Siderophore Conjugation 361 with Daptomycin Confers Potent Activity against Multidrug Resistant Acinetobacter baumannii Both in 362 Vitro and in Vivo. J Med Chem 60, 4577-4583. 
36319 Liu R, Miller PA, Vakulenko SB, Stewart NK, Boggess WC \& Miller MJ (2018) A Synthetic Dual

364 Drug Sideromycin Induces Gram-Negative Bacteria To Commit Suicide with a Gram-Positive Antibiotic.

365 J Med Chem 61, 3845-3854.

36620 Houot L, Fanni A, de Bentzmann S \& Bordi C (2012) A bacterial two-hybrid genome fragment library

367 for deciphering regulatory networks of the opportunistic pathogen Pseudomonas aeruginosa. Microbiol

368 Read Engl 158, 1964-1971.

36921 Battesti A \& Bouveret E (2012) The bacterial two-hybrid system based on adenylate cyclase

370 reconstitution in Escherichia coli. Methods San Diego Calif 58, 325-334.

37122 Gasser V, Baco E, Cunrath O, August PS, Perraud Q, Zill N, Schleberger C, Schmidt A, Paulen A,

372 Bumann D, Mislin GLA \& Schalk IJ (2016) Catechol siderophores repress the pyochelin pathway and

373 activate the enterobactin pathway in Pseudomonas aeruginosa: an opportunity for siderophore-antibiotic

374 conjugates development. Environ Microbiol 18, 819-832.

37523 Kang Y, Zarzycki-Siek J, Walton CB, Norris MH \& Hoang TT (2010) Multiple FadD acyl-CoA

376 synthetases contribute to differential fatty acid degradation and virulence in Pseudomonas aeruginosa.

$377 \quad$ PloS One 5, e13557.

37824 Michel L, González N, Jagdeep S, Nguyen-Ngoc T \& Reimmann C (2005) PchR-box recognition by

379 the AraC-type regulator PchR of Pseudomonas aeruginosa requires the siderophore pyochelin as an

380 effector. Mol Microbiol 58, 495-509.

38125 Hannauer M, Barda Y, Mislin GLA, Shanzer A \& Schalk IJ (2010) The ferrichrome uptake pathway in

382 Pseudomonas aeruginosa involves an iron release mechanism with acylation of the siderophore and

383 recycling of the modified desferrichrome. J Bacteriol 192, 1212-1220.

38426 Hannauer M, Schäfer M, Hoegy F, Gizzi P, Wehrung P, Mislin GLA, Budzikiewicz H \& Schalk IJ

385 (2012) Biosynthesis of the pyoverdine siderophore of Pseudomonas aeruginosa involves precursors with a 386 myristic or a myristoleic acid chain. FEBS Lett 586, 96-101.

38727 Vergnolle O, Xu H \& Blanchard JS (2013) Mechanism and regulation of mycobactin fatty acyl-AMP 388 ligase FadD33. J Biol Chem 288, 28116-28125. 
390 the Pseudomonas aeruginosa RetS sensor domain modulate accessibility to the ligand binding site.

391 Environ Microbiol 12, 1775-1786.

\section{$393 \quad$ Figure legends}

395 Fig. 1. Interaction between FptX and FadD1. (A) Bacterial two-hybrid assay. The fadD1 and fptX genes

396 were cloned into the two-hybrid pUT18C or pKNT25 vectors and corresponding vectors were co397 transformed in DHM1 cells that were spotted on Xgal-IPTG LB agar plates. A blue color reflects interaction

398 between chimeric proteins while white color attests the absence of interaction. RetS was reported to form

399 dimers and is used as a positive control [28]. The experiments were done in triplicate and a representative

400 result is shown. (B) Two-step affinity chromatography. Solubilized bacterial membranes overexpressing

401 FadD1(StrepII) and FptX(His 6 ) proteins were submitted to a two-step affinity chromatography: loading into

402 a StrepTrap HP column (1), elution with desthiobiotin into a HisTrap HP column (2), and final elution with

403 imidazole (3). (C) Immunoblot analysis of the fractions corresponding to the different chromatography steps

404 using anti-His ( $\alpha$-His) and anti-StrepII ( $\alpha$-StrepII) antibodies.

406 Fig. 2. ${ }^{55} \mathrm{Fe}$ distribution in $P$. aeruginosa cell compartments after incubation with $\mathrm{PCH}-{ }^{55} \mathrm{Fe}$. Strains were 407 grown in CAA medium and then incubated in the presence of $200 \mathrm{nM} \mathrm{PCH}-{ }^{55} \mathrm{Fe}$ during $30 \mathrm{~min}$. Afterwards, 408 the cells were then pelleted; the extracellular medium, periplasm, cytoplasm and membrane fractions 409 isolated as described in the Materials and methods, and the amount of ${ }^{55} \mathrm{Fe}$ present monitored. The results 410 are expressed as the percentage of pmol of ${ }^{55} \mathrm{Fe}$ incubated with the cells. The errors bars represent the 411 standard deviation of the mean of three independent experiments.

413 Fig. 3. Amount of $\mathrm{PCH}$ secreted by PAO1 and its corresponding mutants. Cells were grown in CAA medium 414 and PCH was extracted from the growth medium as described in Materials and Methods and its 
415 concentration was determined by measuring the absorbance at $320 \mathrm{~nm}$. The errors bars represent the 416 standard deviation of the mean of six independent experiments. Student's t-tests were performed between 417 data $(* * * * \mathrm{P}<0.0001)$.

418

419 Fig. 4. Architecture of the purified proteins. 33- $\AA$ 3D reconstruction of the FadD1-FptX complex (A), 26$420 \AA$ 3D reconstruction of FptX (B) and 17- $\AA$ 3D reconstruction of FadD1(C) without symmetry applied. The 421 maps are displayed in different views. (D) Fitting of FptX (orange) in the EM volume of the FadD1-FptX 422 complex (grey) without symmetry applied.

423 
A

T25 FEBS Letters

FadD1

FptX RetS
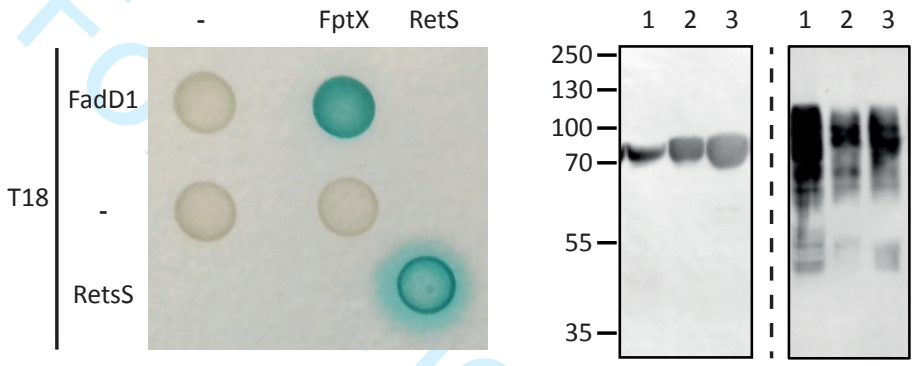

B

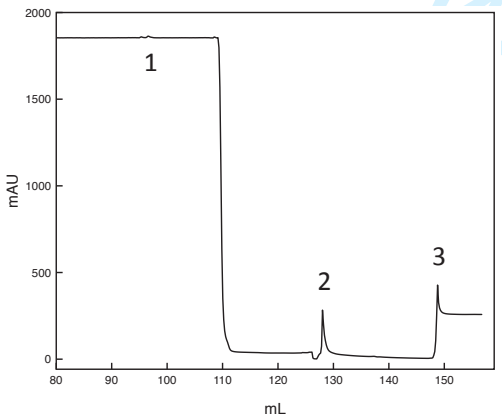


Page 19 of 29 Extracellular medium

Cytoplasm

Membranes

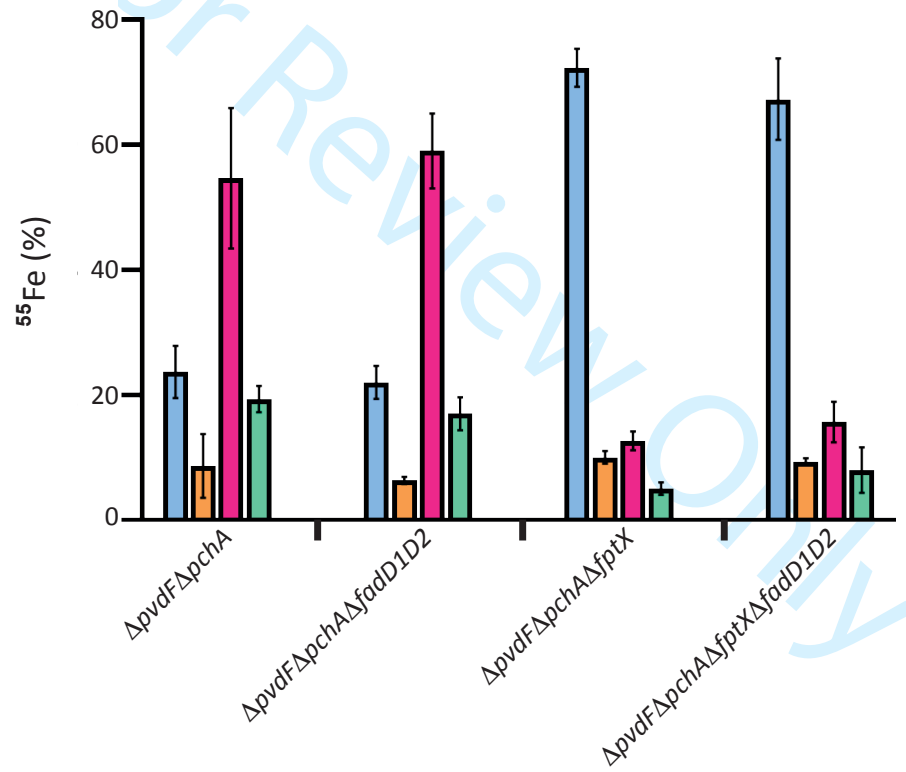


FEBS Letters

Page 20 of 29

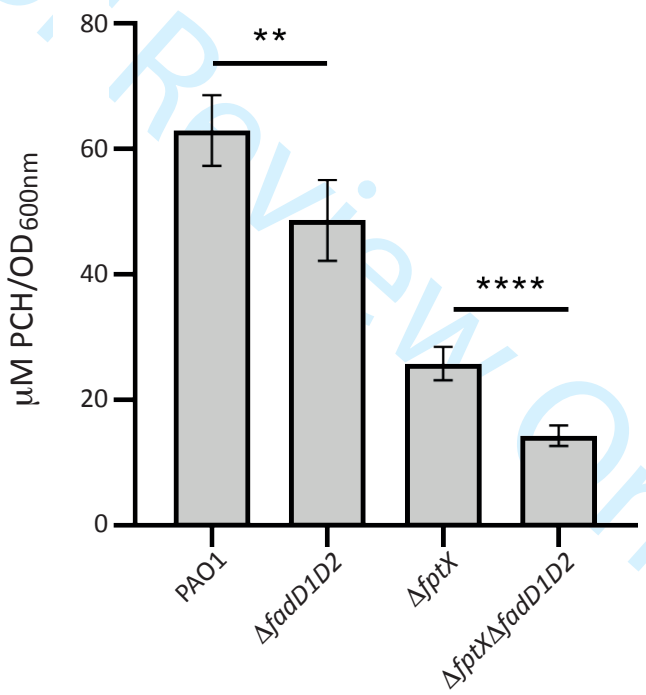


Page 21 of 29

A

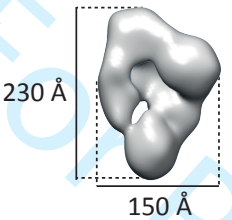

B

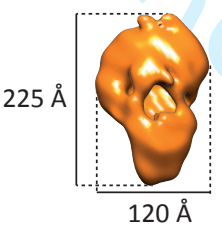

C

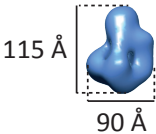

D

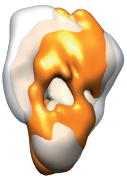

\section{FEBS Letters}
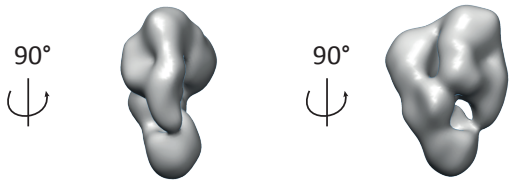

$90^{\circ}$

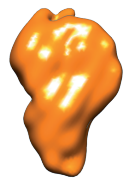

$90^{\circ}$
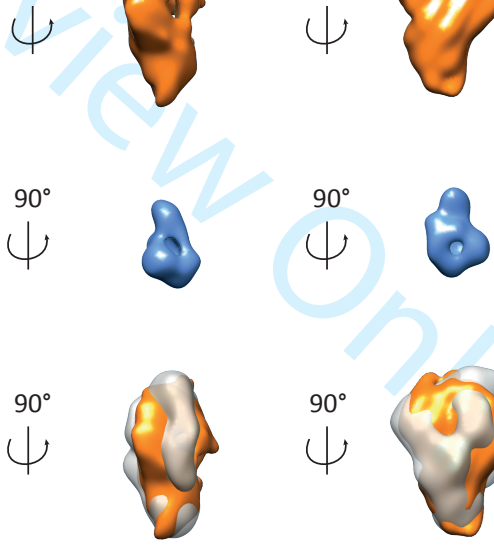

$90^{\circ}$
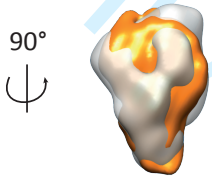


\section{Supplementary data}

3 Identification of the fatty acid coenzyme-A ligase FadD1 as an interacting partner of FptX in the

$4 \quad$ Pseudomonas aeruginosa pyochelin pathway

5

6 Béatrice Roche ${ }^{1,2,3^{*}}$, Gaëtan L.A. Mislin ${ }^{1,2}$ and Isabelle J. Schalk ${ }^{1,2}$

7 1. CNRS, UMR7242, ESBS, Bld Sébastien Brant, F-67412 Illkirch, France.

8 2. Université de Strasbourg, UMR7242, ESBS, Bld Sébastien Brant, F-67412 Illkirch, France.

9 3. Present address: Biozentrum, University of Basel, CH-4056 Basel, Switzerland

$1{ }^{*}=$ correspondence should be addressed to Béatrice Roche (beatrice.roche@unibas.ch)

12 Materials and methods

\section{Binding of $\mathbf{P C H}-{ }^{55} \mathrm{Fe}$.}

$1510 \mathrm{nM}$ of purified FptX or the complex was incubated with various amount of $\mathrm{PCH}-{ }^{55} \mathrm{Fe}$ in $500 \mu \mathrm{L}$ of 50 $16 \mathrm{mM}$ Hepes $\mathrm{pH} 7.5,50 \mathrm{mM} \mathrm{NaCl}, 0.025 \% \mathrm{DDM}$ at room temperature for $2 \mathrm{~h}$. The solution of $\mathrm{PCH}$ 17 complexed with ${ }^{55} \mathrm{Fe}$ was prepared with a siderophore/iron (mol/mol) ratio of 20:1. Incubation was stopped 18 by filtering the assay mixture over GF/B filters (Whatman, presoaked in $0.1 \%$ polyethyleneimine to reduce 19 the non-specific retention). The filters were then rapidly washed three times with $1 \mathrm{~mL}$ of $50 \mathrm{mM}$ Tris $\mathrm{pH}$

208 and counted for radioactivity in a scintillation mixture. The experiment was repeated in the absence of the

21 purified protein. For each $\mathrm{PCH}-{ }^{55} \mathrm{Fe}$ concentration, the non-specific retention was then subtracted from the 22 total radioactivity monitored in the presence of the protein.

\section{Measurement of fatty acyl-CoA synthetase activity of FadD1.}

25 The activity of purified FadD1 was assessed using Ellman's reagent to monitor the consumption of the free 26 thiol group of Co-A due to FadD1-catalyzed acyl-CoA ligation [1]. This colorimetric assay quantifies the 
27 instantaneous free thiol concentration via absorbance readings at $412 \mathrm{~nm}$. A color change is produced upon 28 the binding of 5,5'-dithiobis-(2-nitrobenzoic acid) (DTNB or Ellman's reagent) to the free thiol groups of 29 CoA-SH. Progressive loss of signal at $412 \mathrm{~nm}$ is indicative of CoA-SH consumption. Reactions were 30 prepared with $40 \mu \mathrm{g}$ of purified FadD1 in a reaction buffer containing final concentrations of $150 \mathrm{mM}$ Tris$31 \mathrm{HCl}(\mathrm{pH} 7.2), 10 \mathrm{mM} \mathrm{MgCl} 2,2 \mathrm{mM}$ EDTA, $0.1 \%$ Triton X-100, $5 \mathrm{mM}$ ATP, $0.5 \mathrm{mM}$ co-enzyme A and 32 sodium oleate $(0$ and $200 \mu \mathrm{M})$. To perform the reaction, each mixture was assembled containing all 33 components above (excluding $\mathrm{CoASH}$ ). The reaction mixture was prewarmed at $37^{\circ} \mathrm{C}$ for 2 min. The 34 reaction was initiated with the addition of CoASH (final concentration $0.5 \mathrm{mM}$ ) that was pre-incubated at $3537^{\circ} \mathrm{C}$ for $2 \mathrm{~min}$, quickly mixed, and incubated at $37^{\circ} \mathrm{C}$ during the course of the reaction. $70 \mu \mathrm{L}$ aliquots of 36 the reaction were taken at different intervals and mixed with $600 \mu \mathrm{L}$ of $0.4 \mathrm{mM}$ DTNB (dissolved in $0.1 \mathrm{M}$ 37 potassium phosphate at $\mathrm{pH}$ 8). $\mathrm{A}_{412 \mathrm{~nm}}$ values were acquired after a 5-minute color development period at 38 room temperature. Control reaction mixture lacking protein was also prepared. The previously published 39 extinction coefficient of $13,600 \mathrm{M}^{-1} \cdot \mathrm{cm}^{-1}$ for DTNB was used to compute specific activity [1].

\section{$41 \quad$ Figure legends}

43 Fig. S1. Purification and biochemical characterization of FptX, FadD1 and the FadD1-FptX complex.

44 (A, D and G) Size-exclusion chromatography analysis of the purified FptX (A), FadD1 (D) and the FadD145 FptX complex (G) (continuous line) on a Superdex 200 10/300 GL. The dead volume is indicated as the V0.

46 The arrow indicates the position of the fraction corresponding to FptX (A), FadD1 (D) and the FadD1-FptX 47 complex (G). (B and H) Immunoblot analysis of the purified FptX (B) and the FadD1-FptX complex (H) 48 using anti-His ( $\alpha$-His) and anti-StrepII ( $\alpha$-Strep) antibodies. (C and I) Binding of PCH- ${ }^{55} \mathrm{Fe}$ to purified FptX

49 (C) and FadD1-FptX complex (I). Purified proteins were incubated with 10, 100 or $200 \mathrm{nM}$ of $\mathrm{PCH}-{ }^{55} \mathrm{Fe}$ 50 complex. The non-specific retention was subtracted from the total of radioactivity monitored in the presence

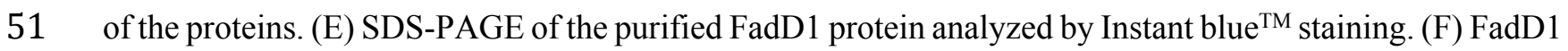

52 enzymatic assay was conducted using DTNB to monitor CoA-SH consumption at $412 \mathrm{~nm}$ with increasing 
53 amount of sodium oleate as substrate. A loss of signal at $412 \mathrm{~nm}$ is indicative of FadD1 activity. The errors

54 bars represent the standard deviation of the mean of three independent experiments.

55

56 Fig. S2. Representative electron micrograph showing FadD1-FptX (A), FptX (D) and FadD1 (G) particles

57 present in several orientations. Scale bar, (A and G) $50 \mathrm{~nm}$, (D) 20nm. Representative views of FadD1-FptX

58 (B), FptX (E) and FadD1 (H). Typical class averages of FadD1-FptX (C), FptX (F) and FadD1 (I).

59

60 Fig. S3. FSC curves of FptX (A), the FadD1-FptX complex (B) and FadD1 (C) reconstruction without

61 symmetry applied. The FSC curves were calculated in Relion using the masked reconstruction of FptX, the

62 FadD1-FptX complex and FadD1, respectively.

63

64

65

66

67

68

69

70

71

72

73

74

75

76

77

78 
79 Table S1. Strains and plasmids used in this study.

\begin{tabular}{|c|c|c|c|}
\hline Strains & & Characteristics & Reference \\
\hline Escherichia coli & ID collection & & \\
\hline TOP10 & 2 & $\begin{array}{l}\text { F-, mcrA } \Delta(\text { mrr-hsdRMS-mcrBC }), \phi 80 l a c Z, \Delta M 15, \Delta \text { lacX74, recA1, } \\
\text { araD139, }(\text { ara-leu }) 7697, \text { galU, galK, rpsL, endA1, mup }\end{array}$ & Invitrogen \\
\hline E. coli DHM1 & 281 & F-, cya-, recA1, endA, gyrA96, thi-1, hsdR17, spoT1, rfbD1, cya-854 & {$[2]$} \\
\hline \multicolumn{4}{|l|}{ Pseudomonas aeruginosa } \\
\hline PAO1 & 43 & Wild-type strain & {$[3]$} \\
\hline$\Delta f p t X$ & PAS218 & PAO1; fpt $X$ chromosomally deleted & [4] \\
\hline$\triangle \mathrm{fadD} 1 \mathrm{D2}$ & PAS431 & PAO1; fadD1 and fadD2 chromosomally deleted & This study \\
\hline$\triangle f p t X \triangle f a d D 1 D 2$ & PAS410 & PAO1; fadD1, fadD2 and $f p t X$ chromosomally deleted & This study \\
\hline$\Delta p v d F \Delta p c h A$ & PAS213 & PAO1; $p v d F$ and $p c h A$ chromosomally deleted & [4] \\
\hline$\Delta p v d F \Delta p c h A \Delta f p t X$ & PAS273 & PAO1; $p v d F, p c h A$ and $f p t X$ chromosomally deleted & {$[4]$} \\
\hline$\triangle p v d F \triangle p c h A \triangle f a d D 1 D 2$ & PAS428 & PAO1; $p v d F, p c h A, f a d D 1$ and $f a d D 2$ chromosomally deleted & This study \\
\hline$\Delta p v d F \Delta p c h A \Delta f p t X \triangle f a d D 1 D 2$ & PAS413 & PAO1; $p v d F, p c h A, f p t X, f a d D 1$ and $f a d D 2$ chromosomally deleted & This study \\
\hline Plasmids & $\begin{array}{l}\text { ID } \\
\text { collection }\end{array}$ & Characteristics & Reference \\
\hline pKNT25 & 264 & P15 origin, Plac, C-terminal T25 cyclase fragment, Kan $^{\mathrm{R}}$ & {$[2]$} \\
\hline pUT18C & 265 & ColE1 origin, Plac, N-terminal T18 cyclase fragment, $\mathrm{Ap}^{\mathrm{R}}$ & {$[2]$} \\
\hline pBAD33 & 270 & pBAD33 plasmid, $\mathrm{Cm}^{\mathrm{R}}$ & [5] \\
\hline pBAD24 & 271 & pBAD24 plasmid, $\mathrm{Ap}^{\mathrm{R}}$ & {$[5]$} \\
\hline pUT18CPAO1lib & 294 & pUT18C containing PAO1 genomic library as a C-terminal T18 fusion & [6] \\
\hline pUT18PAO1lib & 295 & pUT18 containing PAO1 genomic library as an N-terminal T18 fusion & {$[6]$} \\
\hline pUT18+1PAO1lib & 296 & pUT18+1 containing PAO1 genomic library as an N-terminal T18 fusion & {$[6]$} \\
\hline pUT18+2PAO1lib & 297 & pUT18+2 containing PAO1 genomic library as an $\mathrm{N}$-terminal T18 fusion & {$[6]$} \\
\hline pKNT25-fptX & 268 & pKNT25 plasmid containing $f p t X$-cyaAT25 fusion & This study \\
\hline pUT18C-fadD1 & 293 & pUT18C plasmid containing cyaAT18-fadD1 fusion & This study \\
\hline p33-fptX(His $\left.{ }_{6}\right)$ & 272 & pBAD33 plasmid expressing fptX Cter-fused to 6 His tag & This study \\
\hline p24-fadD1(StrepII) & 311 & pBAD24 plasmid expressing fadD1 Cter-fused to StrepII tag & This study \\
\hline pEXG2 delfadD1D2 & 330 & suicide vector for deleting $f a d D 1$ and $f a d D 2$ & This study \\
\hline
\end{tabular}

80

81 Table S2. Oligonucleotides used in this study.

\begin{tabular}{|c|c|l|}
\hline Name & ID collection & \multicolumn{1}{c|}{ Sequence (5' $\rightarrow$ 3') } \\
\hline pKT25-fptXXbaI & 951 & CCGGTCTAGAGATGCTTGAGCTGTACCGCCACCGC \\
\hline pKT25-fptXKpnI & 952 & CCGGGGTACCTCAGGCCTCTTCCGCCCGCCCCTT \\
\hline pKT25-fadD1UP & 1037 & CCGGTCTAGAGATGATCGAAAACTTCTGGAAGGAC \\
\hline pKT25-fadD1DO & 1038 & CCGGGGTACCCGCTTCTGGCCCGCTTTCTTCAGCTC \\
\hline fptX-UPEcoRI & 970 & CCGGGAATTCAAGGAGATATACATATGCTTGAGCTGTACCGCCACCGC \\
\hline fptX-DOHindIII & 969 & CCGGAAGCTTTCAGGCCTCTTCCGCCCGCCCCTT \\
\hline fadD1-UPp24 & 1157 & CCGGCCATGGCCATCGAAAACTTCTGGAAGGACAAG \\
\hline fadD1-DOStrepCter & 1158 & CCGGAAGCTTTCATTTTTCGAACTGCGGGTGGCTCCAGCTAGCCTTCTGGCCCGCTTTC \\
\hline TTCAGCT \\
\hline DelfadD1-500UPHindIII & 1150 & CCGGAAGCTTGCTTCAATGTCTACCCCAACGAGATCGAAG \\
\hline Del fadD1-ATG rev & 1152 & TGGGTACTTGTCCTTCCAGAAGTTTTCGATCAT \\
\hline Del fadD1-STOP for & 1153 & TCTGGAAGGACAAGTACCCACTGCGCGACGAAGAGCTGAAGAAA \\
\hline Del fadD2-500UPHindIII & 1280 & CCGGAAGCTTTTGCCCCAGCCGCCCTGGTCGGCGAAGTGG \\
\hline Del ATG-fadD2 rev & 1281 & TTCAGGTTGCATTTATTCTTGTCCTCTTACCTG \\
\hline Del STOP-fadD1fadD2 for & 1282 & GTAAGAGGACAAGAATAAATGCAACCTGAAAAAGCGGGCCAGAAGTAAGTCCGC \\
\hline
\end{tabular}




\section{References}

84

851 Ellman GL (1959) Tissue sulfhydryl groups. Arch Biochem Biophys 82, 70-77.

862 Karimova G, Dautin N \& Ladant D (2005) Interaction network among Escherichia coli membrane proteins

87 involved in cell division as revealed by bacterial two-hybrid analysis. J Bacteriol 187, 2233-2243.

883 Stover CK, Pham XQ, Erwin AL, Mizoguchi SD, Warrener P, Hickey MJ, Brinkman FS, Hufnagle WO,

89 Kowalik DJ, Lagrou M, Garber RL, Goltry L, Tolentino E, Westbrock-Wadman S, Yuan Y, Brody LL,

90 Coulter SN, Folger KR, Kas A, Larbig K, Lim R, Smith K, Spencer D, Wong GK, Wu Z, Paulsen IT, Reizer

91 J, Saier MH, Hancock RE, Lory S \& Olson MV (2000) Complete genome sequence of Pseudomonas

92 aeruginosa PAO1, an opportunistic pathogen. Nature 406, 959-964.

934 Cunrath O, Gasser V, Hoegy F, Reimmann C, Guillon L \& Schalk IJ (2015) A cell biological view of the

94 siderophore pyochelin iron uptake pathway in Pseudomonas aeruginosa. Environ Microbiol 17, 171-185.

955 Guzman LM, Belin D, Carson MJ \& Beckwith J (1995) Tight regulation, modulation, and high-level

96 expression by vectors containing the arabinose PBAD promoter. $J$ Bacteriol $177,4121-4130$.

976 Houot L, Fanni A, de Bentzmann S \& Bordi C (2012) A bacterial two-hybrid genome fragment library for 98 deciphering regulatory networks of the opportunistic pathogen Pseudomonas aeruginosa. Microbiol Read 99 Engl 158, 1964-1971. 
Rage 27 of 29

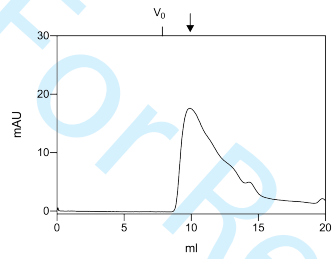

D

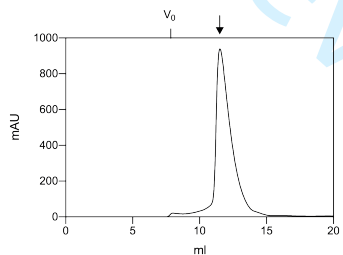

G

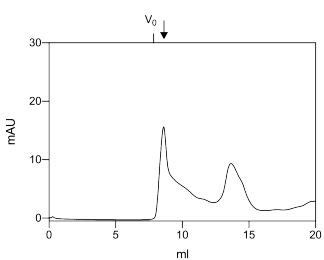

FEBS Letters

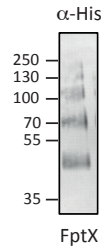

E

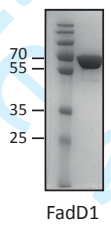

F
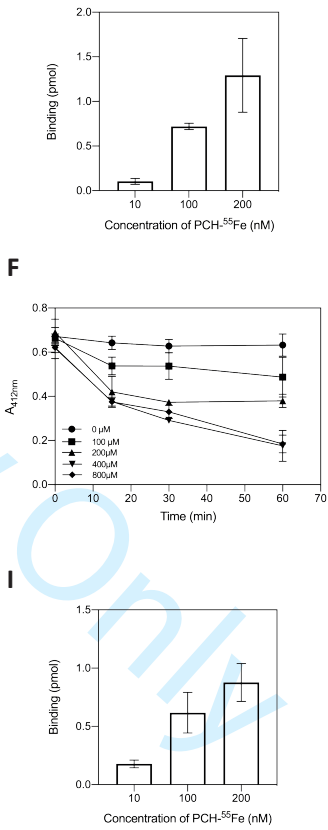

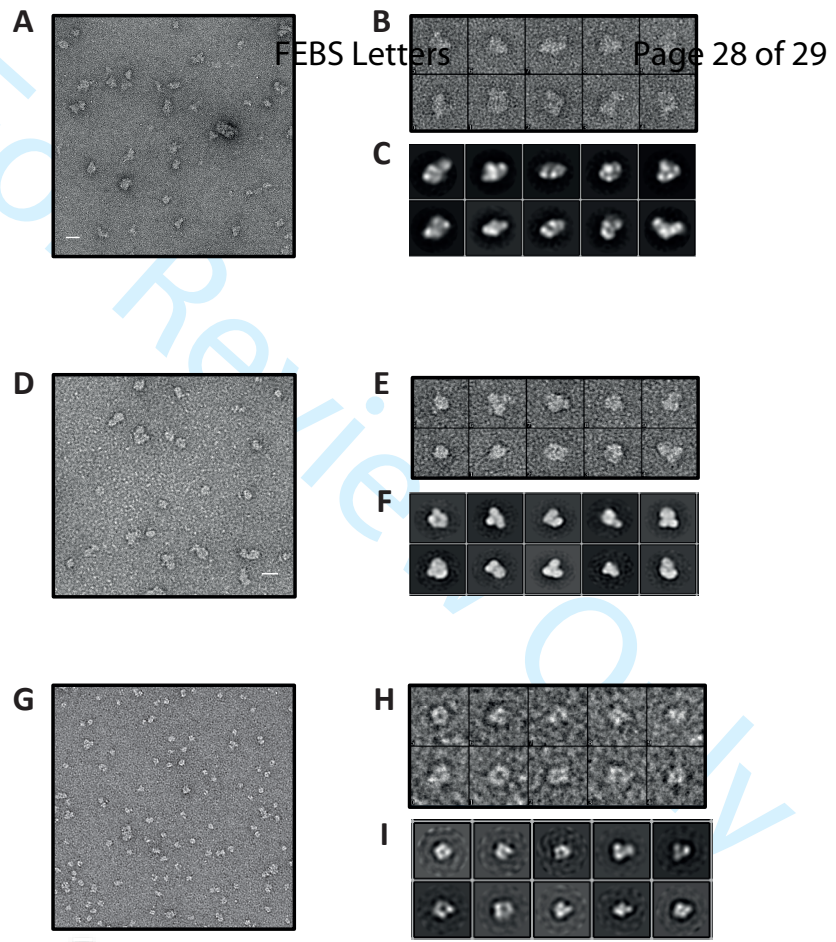


\section{Page 29 of 29}

\section{FEBS Letters}

A

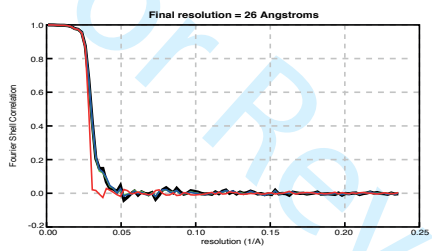

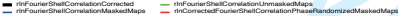

C

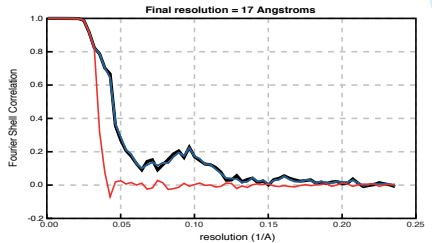

- rhFourierStselicorrelatianCorrected

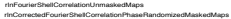

B

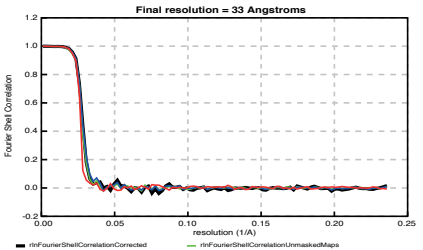

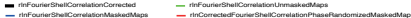

